\title{
$5-23-2014$
}

\section{JPCRR Author Instructions}

Follow this and additional works at: https://aah.org/jpcrr

Part of the Medicine and Health Sciences Commons

\section{Recommended Citation}

JPCRR author instructions. J Patient Cent Res Rev. 2014;1:102-105. doi: 10.17294/2330-0698.1020

Published quarterly by Midwest-based health system Advocate Aurora Health and indexed in PubMed Central, the Journal of Patient-Centered Research and Reviews (JPCRR) is an open access, peer-reviewed medical journal focused on disseminating scholarly works devoted to improving patient-centered care practices, health outcomes, and the patient experience. 


\section{General Information}

Aurora Health Care's Journal of Patient-Centered Research and Reviews (JPCRR) contains original peer-reviewed research and review articles from rigorous scholarly writings of interest to a multidisciplinary audience in the basic and applied health and medical sciences.

$J P C R R$ Mission: To further the ongoing quest for new knowledge by providing a medium for the communication of clinical research, with the purpose of improving the quality of human health, the care of the individual patient and the care of populations.

The first issue was released in Winter 2014. Issues include:

- Original Research • Editorials and Topics

- Systematic Reviews - Specialty Content

- Narrative Reviews

\section{Manuscript Submission}

- To submit a manuscript or to contact the editorial office, email Dennis Baumgardner, Editor-in-Chief, at dennis.baumgardner@aurora.org.

- Submissions must include the following: a cover letter, details regarding the manuscript, the manuscript, any accompanying figure/video files, and email addresses of all authors.

- The submission process is designed so that authors will remain anonymous until the final manuscripts are chosen.

\section{Manuscript Preparation}

\section{Cover Letter}

- Authors must include the title of the manuscript, the manuscript category, and how the manuscript adheres to the objective and mission of JPCRR. Indicate who the corresponding author is (i.e. the author who has primary responsibility for the submission of the manuscript). This person will be notified of decisions and be held responsible for submitting revisions.

\section{Style and Formatting}

- The manuscript should be submitted in Microsoft Word (without macros), double-spaced and formatted with 1" margins and Times New Roman 12 pt. font.

- Text should begin on the third page, following the title page and abstract page.

- Abbreviations should be kept to a minimum and defined when they first appear in the text.

- Heading and subheadings should be clearly denoted.

- Authors must use only one space after periods, not two.

- It is the author's responsibility to present a manuscript free of spelling and grammatical errors.

\section{Title Page}

- The title should reflect the content of the article, be concise and informative.

- Include full names of each author, his/her academic degrees, and his/her current departmental and institutional affiliations and email addresses. Additionally, provide full contact information for the corresponding author, including mailing address, phone number, fax number and email address.

- Keyword bank: please provide a list of 3-5 keywords for the journal's use that are searchable on PubMed.

\section{Conflicts of Interest}

- Authors should provide a list of any and all relationships, circumstances, or conditions that present a potential conflict of interest. This can be included on the title page, either by listing any conflicts of interest or simply stating that none exist.

- If a manuscript is accepted for publication, the author(s) must complete a disclosure form.

\section{Institutional Review Board}

- Original research or any report involving research using human or animal subjects must have Institutional Review Board (IRB) approval prior to submitting. You may be required to provide proof of IRB oversight.

\section{Acknowledgments}

- Authors should list external funding sources and individuals who did not fulfill authorship requirements yet made significant contributions to the manuscript. 


\section{References}

- The corresponding author is responsible for providing accurate and complete references in accordance with AMA style.

- References must be numbered serially in the order in which they appear in the text and typed double-spaced on a separate page.

- Superscripts should always occur after punctuation and, when necessary, embedded in the sentence.

- References appearing in tables/figures should be cited in succession in the text at the location of the table or figure notation. Use Index Medicus (National Library of Medicine) abbreviations for journal titles.

-When citing an article from the Journal of PatientCentered Research and Reviews, it is important to note the correct citation format is $J$ Patient-Centered Res Rev.

- Identify abstracts by inserting the abbreviation (abstr.) after the title.

- List all authors when 6 or fewer; when 7 or more, list first 3 and add "et al." Do not use periods after the authors" initials. Do provide inclusive page numbers. Use the following style and punctuation for citations:

- Citation formats:

- For journals:

ex: Baumgardner DJ, Curtis T, Walters JM, et al. This is an article title. J Patient-Centered Res Rev. 2014;8:429-35.

- For books:

ex: Oh JK, Seward JB, Tajik AJ. The Echo Manual, 3rd Edition. Philadelphia: Lippincott Williams \& Wilkins, 2007, p. 245.

- For chapters:

ex: Mirza M, Shen WK, Jahangir A. Senescence and arrhythmogenesis. In: Gussak I, Antzelevitch C, Wilde AAM, Powell BD, Ackerman MJ, Shen WK (eds). Electrical Diseases of the Heart: Genetics, Mechanisms, Treatment, Prevention, Second Edition. London: Springer-Verlag, 2013, pp. 317-32.

- For online media:

ex: Centers for Disease Control and Prevention. Food safety: preventing the spread of foodborne illness. http://www.cdc.gov/24-7/cdefastfacts/cdefacts. html. Accessed Feb. 20, 2014.

- Unpublished data and personal communications may be cited in the text, but should not be listed as references.

Authors are responsible for the accuracy of their references.

\section{Tables and Figures}

- Generally limit the total number of tables and figures to 10. For Patient-Centered Essays and Creative Writing and inclusion in the From the Other Side of the Table section, we generally only accept one image and no tables.

- Table and figure numbers must correspond with the order they appear in the text.

- Include a title with each table or figure legend. Use superscript lowercase letters in alphabetical order to denote explanatory material in footnotes.

- Format all tables in Microsoft Word and place each on separate pages following the reference list.

-All text within tables should be editable and able to be copied.

- Figures should be submitted electronically as separate TIF or JPG files with minimum resolution of 300 dpi.

- Color figures will appear online at no charge. Authors who request color images in print may be required to financially contribute to printing costs.

\section{Videos}

- Inclusion of videos in the online publication is at the discretion of the editors.

- Video files must be submitted electronically in either AVI or MPG format and should be no larger than 5 megabytes. (It is advisable to compress files to use as little bandwidth as possible.)

- Each video requires a brief descriptive legend, which should be submitted in the main manuscript document on a page following figure legends.

\section{Authorship}

- All authors should follow common ethical standards of research as described in "Uniform Requirements for Manuscripts Submitted to Biomedical Journals."

- Authorship requirements are fulfilled by those who have contributed original ideas, provided data collection and/or analysis, wrote the manuscript, and/or substantially edited the manuscript. All authors must have sufficient knowledge to defend the content of the manuscript.

\section{Permissions}

- Use of previously published tables and figures/images is discouraged unless absolutely necessary for the article. The author is responsible for obtaining permission to reuse material, which can usually be accomplished by contacting the copyright owner. Keep in mind the copyright owner is normally the publisher, not the author. Documentation of permission to reuse previously copyrighted material must accompany the manuscript submission. 


\section{Copyright Agreement}

- Manuscripts are received with the understanding that they are submitted solely to the Journal of Patient-Centered Research and Reviews (JPCRR), that upon submission, they become the property of the Publisher (Aurora Health Care), that the work has not been previously published, and that manuscript data have been reviewed by all authors, who agree with the analyses of the data and the conclusions reached in the manuscript. The Publisher reserves copyright and renewal on all published material, and such material may not be reproduced without the written permission of the Publisher. Statements in articles are the responsibility of the authors.

- If your paper is accepted, all authors must sign our copyright transfer agreement in order to proceed with publication.

\section{Prior presentations}

- Manuscripts will not be considered if they have been previously published, including in both print and electronically. Additionally, the manuscript should not be under consideration elsewhere. If authors have any questions regarding the legitimacy of publication, authors should include copies of what might constitute as a duplicate publication with the submission form. Exceptions may be considered for unusual circumstances.

- Abstracts and other documents published in relation to scientific meetings are acceptable and do not adhere to the restrictions above.

\section{Duplicate Publications}

- Manuscripts will not be considered if they have been previously published, including both in print and electronically. Additionally, the manuscript should not be under consideration elsewhere. If authors have any questions regarding the legitimacy of publication, authors should include copies of what might constitute a duplicate publication along with the submission form. Exceptions may be considered for unusual circumstances.

- Data from abstracts and other documents published in relation to scientific meetings are acceptable and do not adhere to the restrictions above.

\section{Manuscript Categories}

- We do not accept traditional case reports or manuscripts in which a case report is the sole focus. However, an abbreviated case report may be utilized to introduce a narrative or systematic review. Case series are acceptable submissions if the series includes more than 3 cases. We also do not accept letters to the editor. However, we do accept Editorials (see below).

- All articles should pertain to JPCRR's mission statement.

- Conclusion: You need not reiterate what is described in the categories "Results" and "Discussion." However, each manuscript must have a conclusion that includes a final summative statement and explanation of how the content of the manuscript and/or results of the study would potentially benefit the care of a patient.

We accept articles in the following categories:

\section{Original Research}

- Content: Original research from diverse perspectives - clinical, biomedical, behavioral and social sciences are of primary interest. Original and novel manuscripts will receive priority for publication. We accept animal research and negative results articles.

- Format: Introduction, Methods, Results, Discussion, Conclusion.

- Length: Maximum 4,000 words.

- Abstract: No more than 250 words. Structured with paragraphs labeled: Purpose, Methods, Results, Conclusion.

\section{Systematic Review}

- Content: A critical assessment and evaluation of all relevant research studies that address a particular clinical issue. Systematic reviews should include a description of the findings of the collection of research studies and a meta-analysis (if applicable).

- Format: Introduction, Methods, Results, Discussion, Conclusion.

- Length: Maximum of 4,000 words.

- Abstract: No more than 250 words. Structured with paragraphs labeled: Purpose, Methods, Results, Conclusion.

\section{Narrative Review}

- Content: A review and synopsis of pertinent, current (and historical when appropriate) literature on a specific topic. Selected literature should provide a fair representation of the current literature.

- Format: Introduction, Methods, Results, Discussion, Conclusion.

- Length: Maximum of 4,000 words.

- Abstract: No more than 250 words. Unstructured. 


\section{Editorials and Topics}

1. Invited Editorial

- Content: Concurrent scholarly opinion piece in which the author discusses a specific topic or article in the journal, contributing to basic and applied health and medical care. You must be invited to write this particular type of editorial. We do not accept letters to the editor.

- Length: Maximum of 2,000 words.

- Abstract: No more than 250 words. Unstructured.

2. Topic Synopsis

- Content: Scholarly opinion piece or evidence-based synopsis that discusses pertinent issues pertaining to basic and applied health and medical care. These do not necessarily refer to a particular article published in the journal. You may write this type of editorial or synopsis without an invitation.

- Length: Maximum of 2,000 words.

- Abstract: No more than 250 words. Unstructured.

\section{Specialty Content}

Content: This category is reserved for manuscripts that do not adhere to the other categories, yet significantly contribute to medical knowledge, intellectual discussion and patient-centered health.

1. Clinical (Grand) Rounds

- Content: Submissions in which health care professionals discuss the clinical decision-making process in conversational format. These should provide a fair representation of a particular subject by relying on generally recent information and a comparison of two or more of the alternative modalities. This is a reference-based discussion, not a simple opinion piece. Include a Discussion and Conclusion in your submission.

- Length: Maximum of 4,000 words.

- Abstract: No more than 250 words. Unstructured.

2. Medical Education

- Content:Articles pertaining to specific topics of medical education that do not otherwise fit into previously mentioned categories.

- Length: Maximum of 2,000 words.

- Abstract: No more than 250 words. Unstructured.
3. Patient-Centered Essays and Creative Writing

- Content: Any form of creative writing (essays, poems, short stories, etc.) pertaining to his/her experience or interaction with a patient or groups of patients. People of all health care disciplines are welcome to submit. We will only accept writing from health care professionals for this section. (See "From the Other Side of the Table" section).

- Length: Maximum of 2,000 words for essays, 900 words for poems, and 4,000 words for short stories.

- Abstract: No more than 150 words. Unstructured for all essays except for poems, which should not include abstracts.

4. From the Other Side of the Table

- Content: Clinical authors may submit essays from the patient's perspective either because they were a patient or a loved one was a patient. Alternatively, patients who are not health care workers also may submit.

- Length: Maximum of 2,000 words.

- Abstract: No more than 250 words. Unstructured.

\section{Editorial Freedom}

- The journal does not endorse companies, products or services displayed in any advertisements.

- Decisions of the Editorial Board are final.

\section{Peer Review Process and Revisions}

- Each manuscript will receive a minimum of two anonymous reviews in addition to the editorial review.

- Most manuscripts require revisions. Corresponding authors will be given editorial decisions and feedback. Authors are responsible for completing these revisions in a timely manner, as prescribed by the response from the editors. 